\title{
Effect of rutin from tartary buckwheat sprout on serum glucose-lowering in animal model of type 2 diabetes
}

\author{
DONG-GI LEE ${ }^{1 *}$ \\ IK SOON JANG ${ }^{2 *+}$ \\ KYEONG EUN YANG ${ }^{1}$ \\ SO-JUNG YOON ${ }^{1}$ \\ SUJEONG BAEK ${ }^{3}$ \\ JOO YONG LEE ${ }^{3}$ \\ TATSURO SUZUKI ${ }^{4}$ \\ KEUN-YOOK CHUNG 5 \\ SUN-HEE WOO ${ }^{6 *}$ \\ JONG-SOON CHOI $1,3 *$ \\ ${ }^{1}$ Biological Disaster Analysis Group, Korea Basic \\ Science Institute, Daejeon 34133, Republic of Korea \\ ${ }^{2}$ Drug \& Disease Target Group, Korea Basic Science \\ Institute, Daejeon 34133, Republic of Korea \\ ${ }^{3}$ Graduate School of Analytical Science and Technology \\ Chungnam National University, Daejeon 34134 \\ Republic of Korea \\ ${ }^{4}$ National Agricultural Research Center for Hokkaido \\ Region, Memuro, Hokkaido 0820081, Japan \\ ${ }^{5}$ Department of Agricultural Chemistry, Chungbuk \\ National University, Cheongju 28644, Republic \\ of Korea \\ ${ }^{6}$ Department of Crop Science, Chungbuk National \\ University, Cheongju 28644, Republic of Korea
}

Accepted January 11, 2016

Published online April 8, 2016

\begin{abstract}
This study investigates the anti-diabetic effects of rutin from tartary buckwheat sprout in type 2 diabetes mouse model. The rutin content in tartary buckwheat sprout (TBS) is five times higher than that found in common buckwheat sprout (CBS) as evident from high-performance liquid chromatography analysis. Administration of either rutin or TBS ethanolic extract to diabetes mice decreased the serum glucose level significantly. Rutin down-regulated the expression levels of protein-tyrosine phosphatase $1 \mathrm{~B}$; it is negative regulator of insulin pathway, both transcriptionally and translationally in myocyte $\mathrm{C} 2 \mathrm{C} 12$ in a dose-dependent manner. In conclusion, rutin can play a critical role in down-regulation of serum glucose level in type 2 diabetes.
\end{abstract}

Keywords: tartary buckwheat sprout, rutin, type 2 diabetes, insulin signaling

Buckwheat is a nutritious and pharmaceutically important plant. It contains valuable components that protect human health against inflammation and carcinogenesis (1). Among the main components, rutin, namely rutoside, flavonoid glycoside, is one of the most abundant polyphenols in tartary buckwheat (2). In particular, the amount of rutin has been known to be abundant in buckwheat sprout leaves. Use of buckwheat has

\footnotetext{
* These authors contributed equally

${ }^{+}$Current address; Biological Disaster Analysis Group, Korea Basic Science Institute, Daejeon 34133, Republic of Korea

*Correspondence; e-mail: shwoo@chungbuk.ac.kr, jschoi@kbsi.re.kr
} 
been well known in the management of diabetes mellitus. So far, the antidiabetic effect of buckwheat was attributed to $D$-chiro inositol as a source of insulin mediator in buckwheat seed bran (3). In addition, the seed of tartary buckwheat was reported to improve high glucose-induced insulin resistance and diabetes in mice (4). Protein-tyrosine phosphatase $1 \mathrm{~B}(\mathrm{PTP1B})$ is known to be a negative regulator of the insulin signaling pathway. Increased expression of PTP1B can regulate the downstream insulin signal transduction, leading to the inhibition of glucose uptake and glycogen synthesis (5). However, there are only few studies about rutin from buckwheat sprouts against diabetes. Thus, we focused on the antidiabetic effects of rutin from tartary buckwheat sprout. The purpose of the present study was to investigate whether the administration of either tartary buckwheat sprout ethanol extract or rutin lowered the serum glucose levels in vivo. In addition, we examined the mechanism of action of rutin with the target gene expression involved in insulin signaling in preadipocyte and myocyte cell lines by reverse transcriptase-polymerase chain reaction (RT-PCR) and immunoblot analysis.

\section{EXPERIMENTAL}

\section{Materials}

Common buckwheat sprout (CBS) (Fagopyrum esculentum) and tartary buckwheat sprout (TBS) (Fagopyrum tartaricum) were obtained from the farm of the Chungbuk National University (Cheongju, Republic of Korea). Rutin (purity $\geq 95 \%$ ), isoorientin, vitexin, isovitexin and quercetin were purchased from Sigma (USA). Mouse cell lines 3T3-L1 and C2C12 were obtained from the American Type Culture Collection (USA). Male C57BL/6J and diabetes C57BL/6J (B6.Cg-m ${ }^{+/+}$eepr $^{\mathrm{db} / \mathrm{J}}$ ) mice were kindly provided by the Korea Research Institute of Bioscience and Biotechnology (Republic of Korea). C57BL/6J mouse is a common inbred strain with a dark brown coat. The homozygous spontaneous mutation (B6.Cg-m ${ }^{+/+} \mathrm{Lepr}^{\mathrm{db} / \mathrm{J}}$ ) was used for diabetes mouse ( $d b / d b$ mouse). Mice were fed a normal diet ad libitum and were kept in a temperature- and humidity-controlled standard animal house cage with 12 hour light/dark cycle. Animal treatment was approved by the animal care protocol of the Korea Basic Science Institute (Daejeon, Republic of Korea) review committee.

\section{Analysis of phenolic compounds from buckwheat sprouts}

Extraction of buckwheat sprouts and analysis of phenolic compounds from CBS and TBS were performed as described previously (6). Standard chemicals such as rutin, isoorientin, vitexin, isovitexin and quercetin were applied under the same high-performance liquid chromatography (HPLC) conditions, and the retention times of buckwheat sprout extract components were compared to those of the standards.

\section{Intravenous glucose tolerance test}

Tester control and $d b / d b$ mice were fasted overnight. For intravenous glucose tolerance test, rutin $(1 \mathrm{mg})$ or TBS extract $(10 \mathrm{mg})$ was dissolved in $25 \mu \mathrm{L}$ dimethyl sulfoxide (5\%, $V / V)$ and $75 \mu \mathrm{L}$ of distilled water added to dimethyl sulfoxide dilution. The solution of rutin ( $1 \mathrm{mg}$ per $100 \mu \mathrm{L}$ or $10 \mathrm{mg}$ per $\mu \mathrm{L}$ TBS) was administered to the oral cavity 2 hours before glucose injection $(n=3)$. The first blood sample was collected from the tail vein at 
the start time ( $0 \mathrm{~min})$ and, immediately, a $5 \%$ glucose solution ( $2 \mathrm{~g}$ per $\mathrm{kg}$ body mass) was injected to the tail vein. Blood was consecutively collected every $30 \mathrm{~min}$ up to $180 \mathrm{~min}$. Serum glucose level was assessed using a portable glucose oxidase digital assay kit (LifeScan, Johnson \& Johnson, USA).

\section{Cell culture, reverse-transcriptase PCR and immunoblot analyses}

Mouse preadipocyte (3T3-L1) and myocyte cell line (C2C12) were planted into 6-well plates and maintained in Dulbecco's modified Eagle's medium (DMEM) supplemented with $10 \%(V / V)$ fetal bovine serum at $37^{\circ} \mathrm{C}$ in a $5 \%(V / V) \mathrm{CO}_{2}$ incubator. Cultured cells were pretreated with rutin $\left(100\right.$ and $\left.250 \mathrm{mmol} \mathrm{L}^{-1}\right)$. After one-day treatment, the cell growth was stopped and total RNA was isolated with TriZol (Invitrogen, USA) and RNasefree DNase (Takara, Japan), according to the manufacturers' instructions. The total RNA quantification and cDNA synthesis were performed as described previously (7). The relative mRNA expression level of protein tyrosine phosphatase 1B (PTP1B) was examined with a specific primer pair (sense: 5'-TGG GAA CTG GGC GGC TAT TAC-3', antisense: 5'TCC ACT GAT CCT GCA CTG ACG AG-3'). The primer for glyceraldehyde 3-phosphate dehydrogenase (GAPDH) (sense: 5'-CAT CTC TGC CCC CTC TGC T-3', antisense: 5'-ACG CCT GCT TCA CCA CCT T-3') was used as internal control. Protein expression was examined by immunoblot analysis according to Hwang et al. (8). Protein concentrations of cell lysates were quantified using a bicinchoninic acid protein assay kit (Thermo Fisher Scientific, USA) employing bovine serum albumin as the standard according to the manufacturer's instructions. An equal amount of total protein $(30 \mu \mathrm{g})$ was separated by $12 \%$ sodium dodecyl sulfate polyacrylamide gel electrophoresis (SDS-PAGE) and transferred to a nitrocellulose membrane. The membrane was subsequently incubated with a primary antibody against PTP1B (\#5311, Cell Signaling Technology, USA) or/and anti-GAPDH (\#SC25778, Santa Cruz Biotechnology, USA) as internal control at the dilution level of 1:4,000 overnight. Horseradish peroxidase conjugated-goat anti-rabbit IgG secondary antibody was used for immunodetection at the dilution level of 1:2,000 or 1:8,000 for PTP1B and GAPDH, respectively. The immunoblot signals were detected using enhanced chemiluminescence (Millipore, USA) and visualized on X-ray films (Kodak, USA)

\section{RESULTS AND DISCUSSION}

Phenolic compounds in the ethanol extracts of CBS and TBS were analyzed by reverse phase-HPLC as described previously (9). The chromatograms of CBS and TBS extracts are shown in Fig. 1. Several phenolic compounds, such as isoorientin $\left(t_{\mathrm{R}}=25 \mathrm{~min}\right)$, vitexin $\left(t_{\mathrm{R}}=28\right.$ $\mathrm{min}$ ) and isovitexin $\left(t_{\mathrm{R}}=32 \mathrm{~min}\right)$ were identified in CBS besides rutin $\left(t_{\mathrm{R}}=30.5 \mathrm{~min}\right)$ and quercetin $\left(t_{\mathrm{RT}}=43 \mathrm{~min}\right)$ whereas rutin $\left(t_{\mathrm{R}}=31 \mathrm{~min}\right)$ was predominantly observed in TBS. The chromatogram patterns of buckwheat flavonoids were very similar to the previous report (5). The content of rutin in CBS and TBS was $11 \mathrm{mg}$ and $50 \mathrm{mg}$ per gram dry mass, respectively. Thus, the amount of rutin in TBS was approximately 5 times higher than that in CBS. Highly concentrated rutin in buckwheat leaves was in good agreement with other plant parts $(10,11)$.

To evaluate the lowering effects of serum glucose after oral administration, either rutin $(1 \mathrm{mg}$ per $100 \mu \mathrm{L}$ ) or TBS extract $(10 \mathrm{mg}$ per $100 \mu \mathrm{L})$ was applied to the control and $d b /$ $d b$ mice, followed by detection of the serum glucose level. As shown in Figs. $2 a$ and $b$, the oral pre-treatment with rutin showed no significant difference at time 0 , whereas rutin 

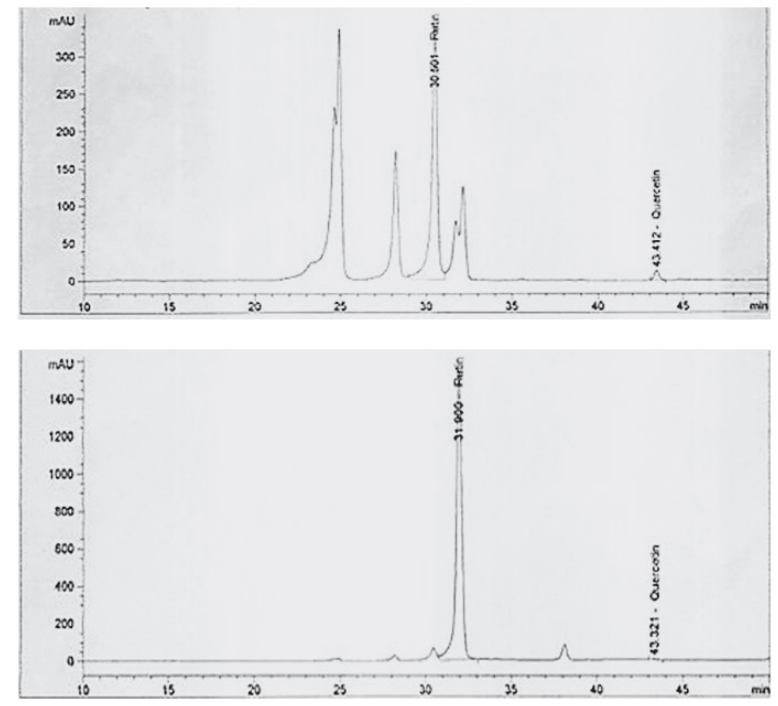

Fig. 1. HPLC chromatogram of ethanol extracts from: a) CBS and b) TBS.

suppressed the serum glucose level after $30 \mathrm{~min}(p<0.05)$. Rutin extract gradually decreased serum glucose level in $d b / d b$ mice after oral administration (Fig. $2 b$ ). When TBS was applied to control mice, the serum glucose level decreased compared to the control group within $60 \mathrm{~min}$ (Fig. 2a) $(p<0.05)$. In $d b / d b$ mice, pre-treatment with TBS showed a similar effect as that of rutin up to $120 \mathrm{~min}$ (Fig. 2b) $(p<0.05)$. This result indicates that rutin has the capacity of serum glucose level lowering, and is suggested as a therapeutic compound in type 2 diabetes. Likewise, an insulin mediator, D-chiro-inositol abundant in buckwheat, was reported to reduce the serum glucose level in type 1-diabetic rats (3). However, this direct effect of rutin from tartary buckwheat sprouts against type 2 diabetes was not addressed in the glucose tolerance test.
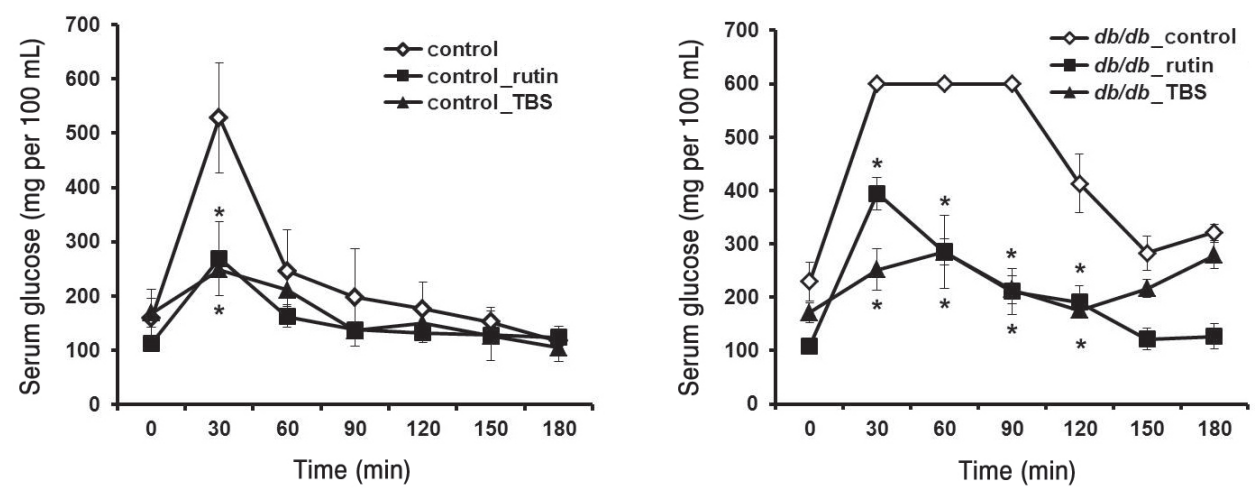

Fig. 2. Intravenous glucose tolerance test in: a) control and b) $d b / d b$ mice (mean $\pm \mathrm{SD}, n=3$ ), ${ }^{*} p<0.05$. 


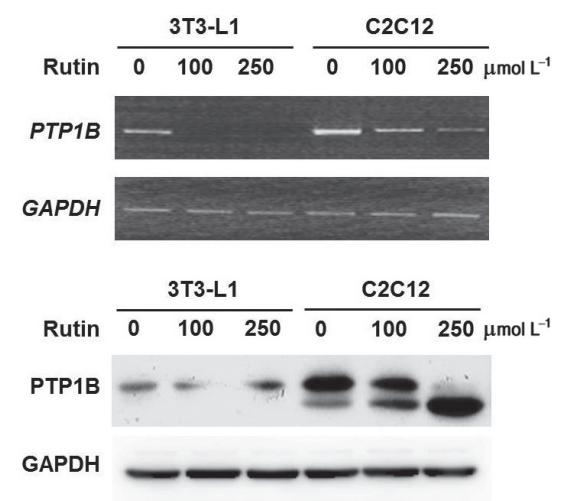

Fig. 3. a) mRNA and b) protein expression of protein tyrosine phosphatase 1B (PTP1B) in 3T3-L1 and $\mathrm{C} 2 \mathrm{C} 12$.

To explore the action mode of rutin in insulin signaling, the gene expression of PTP1B in preadipocyte 3T3-L1 and myocyte C2C12 was examined. Under rutin treatment, the mRNA levels of PTP1B in both cell lines were clearly decreased in a dose-dependent manner, as shown in Fig. 3a. In addition, the protein levels of PTP1B were not so much changed in 3T3-L1 but clearly decreased as the concentration of rutin increased to $250 \mathrm{mmol} \mathrm{L}^{-1}$ in $\mathrm{C} 2 \mathrm{C} 12$ (Fig. 3b). These results suggest that the treatment with rutin activates the insulin receptor downstream signaling by suppressing transcriptionally and translationally the expression of PTP1B. The down-regulation of PTP1B by rutin may improve the insulin resistance and prevent the type 2 diabetic progression in skeletal muscle. Likewise, rutin from buckwheat could preserve the insulin secretory machinery and stimulate the insulin receptor substrate 2 signaling and AMP-kinase in rat pancreatic beta cells (12).

\section{CONCLUSIONS}

Biochemical analysis of serum glucose lowering by rutin was investigated in in vivo and in vitro systems. The serum glucose levels were decreased after $30 \mathrm{~min}$ by rutin pretreatment in $d b / d b$ mice. The rise of serum glucose levels was suppressed in normal mice by rutin pre-treatment at the early time stage $(30 \mathrm{~min})$. When the pre-treatment of TBS extract was applied to the mice, the ability to decrease the serum glucose level was similar to that of rutin for both normal and diabetes mice. mRNA and protein expression of PT$\mathrm{P} 1 \mathrm{~B}$, which is known as negative regulator of insulin receptor signaling pathway, were inhibited in myocyte-derived $\mathrm{C} 2 \mathrm{C} 12$ cell by rutin treatment in a dose-dependent manner. Rutin is an effective compound for lowering serum glucose concentrations, which contributes to the regulation of the insulin signaling pathway in type 2 diabetes mellitus.

Acknowledgements. - This work was supported by a Korea Basic Science Institute project (T35780) to J. S. Choi and supported by the National Research Foundation of Korea Grant funded by the Korean Government (MSIP) (2015, R\&D Equipment Engineer Education Program, 2014R1A6A9064166).

Codes and acronyms. - 3T3-L1 - mouse preadipocyte cell line, AMP - adenosine monophosphate, $\mathrm{C} 2 \mathrm{C} 12$ - mouse myoblast cell line, CBS - common buckwheat sprout, $d b / d b$ mouse, diabetes mouse, GAPDH - glyceraldehyde 3-phosphase dehydrogenase, PTP1B - protein-tyrosine phosphatase 1B, RT-PCR - reverse transcriptase-polymerase chain reaction, TBS - tartary buckwheat sprout. 


\section{REFERENCES}

1. S. T. Ishii, C. Katsumura, K. Shiozuka, K. Ooyauchi, S. Kawasaki, T. Takigawa, Y. Fukushima, M. Tokuji, M. Kinoshita, M. Ohinishi, K. Kawahara and K. Ohba, Anti-inflammatory effect of buckwheat sprouts in lipopolysaccharide-activated human colon cancer cells and mice, Biosci. Biotech. Biochem. 72 (2008) 3148-3157; DOI: 10.1271/bbb.80324.

2. N. Fabjan, I. J. Rode, Z. Kosir, Z. Wang, I. Zhang and I. Kreft, Tartary buckwheat (Fagopyrum tataricum Gaertn.) as a source of dietary rutin and quercetin, J. Agr. Food Chem. 51 (2003) 6452-6455; DOI: 10.1021/jf034543e.

3. J. M. Kawa, C. G. Taylor and R. Przlybylski, Buckwheat concentrate reduces serum glucose in streptozotocin - diabetic rats, J. Agr. Food Chem. 51 (2003) 7287-7291; DOI: 10.1021/jf0302153.

4. C.-C. Lee, W.-H. Hsu, S.-R. Shen, Y.-H. Cheng and S. C. Wu, Fagopyrum tataricum (buckwheat) improved high-glucose-induced insulin resistance in mouse hepatocytes and diabetes in fructoserich diet-induced mice, Exp. Diabetes Res. 2012, 375673; DOI: 10.1155/2012/375673.

5. K. H. Egawa, S. Maegawa, K. Shimizu, Y. Morino, M. Nishio, A. T. Bryer-Ash, J. K. Cheung, R. Kolls, A. Kkiawa and A. Kashiwagi, Protein tyrosine phosphatase-1B negatively regulates insulin signaling in L6 myocytes and Fao hepatoma cells, J. Biol. Chem. 276 (2002) 10207-10211; DOI: 10.1074/jbc. M009489200.

6. C. L. Liu, Y. S. Chen, J. H. Yang and B. H. Chiang, Antioxidant activity of tartary (Fargopyrum tataricum L. Gaertn.) and common (Fargopyrum esculentum Moench) buckwheat sprouts, J. Agr. Food Chem. 56 (2008) 173-178; DOI: 10.1021/jf072347s.

7. J. H. Nam, D.-G. Lee, J. Kwon, C.-W. Choi, S. W. Park, S.-O. Kwon, J. H. Jung, H. C. Park, B. Y. Park, I. S. Jang, W. Y. Bang, C. W. Kim and J.-S. Choi, Comparative proteome analysis of porcine Longissimus dorsi on the basis of pH24 of post-mortem muscle, J. Agr. Sci. 4 (2012) 48-56; DOI: 10.5539/jas. $\mathrm{v} 4 \mathrm{n} 9 \mathrm{p} 48$.

8. J. W. Hwang, Y. M. Baek, K. E. Yang, H. S. Yoo, C. K. Cho, Y. W. Lee, J. Park, C. Y. Eom, Z. W. Lee, J. S. Choi and I. S. Jang, Lactobacillus casei extract induces apoptosis in gastric cancer by inhibiting NF$\kappa$ B and mTOR-mediated signaling, Integr. Cancer Ther. 12 (2012) 165-173; DOI: 10.1177/1534735412442380.

9. L. Bramati, F. Aquilano and P. Pietta, Unfermented rooibos tea: quantitative characterization of flavonoids by HPLC-UV and determination of the total antioxidant activity, J. Agr. Food Chem. 51 (2003) 7472-7474; DOI: 10.1021/jf0347721.

10. I. Kreft, N. Fabjan and K. Yasumoto, Rutin content in buckwheat (Fagopyrum esculentum Moench) food materials and products, Food Chem. 98 (2006) 508-512; DOI: 10.1016/j.foodchem.2005.05.081.

11. I. Musallam, M. Duwayri, R. Shibli and F. Alali, Investigation of rutin content in different plant parts of wild caper (Capparis spinosa L.) populations from Jordan, Res. J. Med. Plant 6 (2012) 27-36; DOI: 10.3923/rjmp.2012.27.36.

12. E. P. Cai and J. K. Lin, Epigallocatechingallate (EGCG) and rutin suppress the glucotoxicity through activating IRS2 and AMPK signaling in rat pancreatic beta cells, J. Agr. Food Chem. 57 (2009) 98179827; DOI: 10.1021/jf902618v. 